\title{
Synthesis and evaluation of antiviral, antitubercular and anticancer activities of some novel thioureas derived from 4-aminobenzohydrazide hydrazones
}

\author{
Pelin Çıkla1, Ş. Güniz Küçükgüzel1', İlkay Küçükgüzel1', Sevim Rollas', \\ Erik De Clercq ${ }^{2}$, Christophe Pannecouque ${ }^{2}$, Graciela Andrei², Robert Snoeck2, \\ Fikrettin Şahin ${ }^{3}$, Ömer Faruk Bayrak ${ }^{3}$
}

\begin{abstract}
A series of novel 1-[4-[[2-[(4-substituted phenyl)methylene]hydrazino]carbonyl]p henyl]-3-substituted thiourea derivatives have been synthesized by the addition of substituted aryl isothiocyanates to 4-amino- $\mathrm{N}^{\prime}$-[(4-substituted phenyl) methylene] benzohydrazide, which was prepared by condensation of 4-aminobenzoic acid hydrazide with 4-fluorobenzaldehyde or 4-(trifluoromethyl)benzaldeyde. All synthesized compounds were evaluated in vitro against HIV-1 (IIIB) and HIV-2 (ROD) strains in MT-4 cells, as well as other selected viruses such as HSV-1, HSV-2, Coxsackie virus B4, Sindbis virus, human cytomegalovirus, and varicella-zoster virus using HeLa, Vero, or HEL cell cultures. Antimycobacterial activity against Mycobacterium tuberculosis $\mathrm{H} 37 \mathrm{Rv}$ was also evaluated. The anticancer activity and cytotoxicity screening of the synthesized compounds were determined on A 549 and L 929 cell lines.
\end{abstract}

KEY WORDS: Hydrazones, Thioureas, Antiviral activity, Anticancer activity, Mycobacterium tuberculosis H37Rv

\section{INTRODUCTION}

Thiacetazone which possesses a thiosemicarbazone structure, has been reported as a tuberculostatic agent (1). Thiocarlide (N,N'-bis[p-(isoamyloxy) phenyl]-thiourea) is known as a potent inhibitor of Mycobacterium tuberculosis (2). N-DAldopentofuranosyl-N'-[ $p$-(isoamyloxy)phenyl] thiourea derivatives, designed as structural analogues of thiocarlide, have recently been reported to be more potent than thiocarlide itself (3). Methisazone was one of the first antiviral compounds used in clinical practice (4) (Figure 1). This drug plays an important role as a prophylactic agent against several viral diseases. Antitubercular effects have been shown with various 4aminobenzoic acid substituted benzalhydrazones (5). Sriram and co-workers have recently reported antitubercular activity of several thiourea derivatives obtained from isonicotinoyl hydrazone (6). Antitumor $(7,8)$ and antitubercular (9-11) activities of some hydrazide-hydrazones and thioureas have been reported. In addition, some thiou- rea derivatives were reported to be potent inhibitors of influenza virus neuraminidase, Coxsackie $\mathrm{B} 4$ virus and thymidine kinase positive varicellazoster virus (TK+ VZV, OKA strain) $(12,13)$.

As a continuation of our previous efforts on 4aminobenzoic acid hydrazones (14) and several thiourea derivatives $(15,16)$, a series of novel thioureas, in which hydrazide-hydrazone and disubstituted thiourea moieties were incorpora ted in one structure, have been synthesized starting from 4-amino- $N^{\prime}$-[[4-fluoro/4-(trifluoromethyl) phenyl] methylene] benzohydrazide and evaluated of their antitubercular, antiviral and anticancer potency. All synthesized compounds were screened in vitro against HIV-1 (IIIB) and HIV-2 (ROD) strains in MT-4 cells, as well as other selected viruses such as HSV-1, HSV-2, Coxsackie B4 virus, Sindbis virus, cytomegalovirus (CMV) and varicella-zoster virus (VZV) using HeLa, Vero or human embryonic lung (HEL) cells.
AFFILIATIONS

1 Marmara University, Faculty of Pharmacy, Department of Pharmaceutical Chemistry, İstanbul, Türkiye

2Katholieke Universiteit Leuven, Rega Institute for Medical Research, Leuven, Belçika

3Yeditepe University, Faculty of Engineering and Architecture, Genetics and Bioengineering Department, İstanbul, Türkiye

CORRESPONDENCE

Ş. Güniz Küçükgüzel

E-mail:gkucukguzel@ marmara.edu.tr

Received:

August 31, 2009

Revision:

October 01, 2009

Accepted:

October 08, 2009 


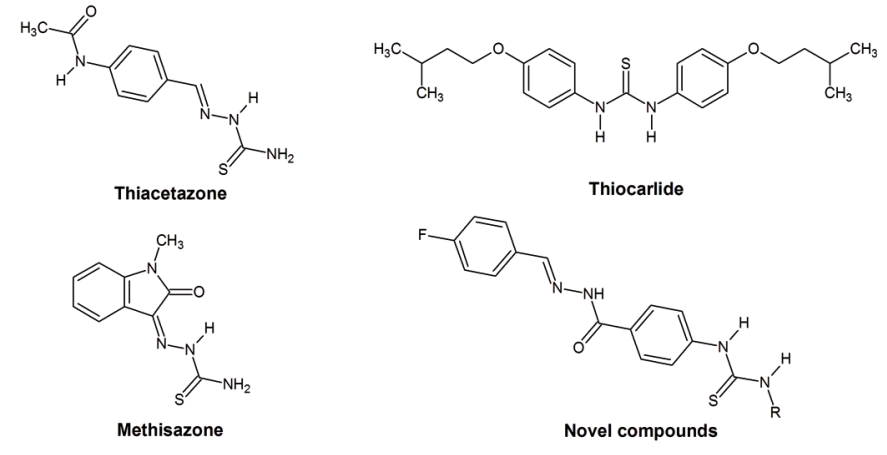

FIGURE 1. Some thioureas with antimycobacterial or antiviral activity.

In vitro antitubercular activity of novel compounds against Mycobacterium tuberculosis H37Rv was evaluated at TAACF. Anticancer potential of the synthesized compounds was determined using the A 549 and L 929 cell lines.

\section{RESULTS AND DISCUSSION \\ Chemistry}

4-Aminobenzoic acid hydrazide $\mathbf{1}$ was prepared by the reaction of ethyl 4-aminobenzoate with hydrazine-hydrate. 4-Amino- $N^{\prime}$-[[4-fluoro/4-(trifluoromethyl)phenyl]methylene]-benzohydrazide 2-3 (cf. Experimental Section) were synthesized by condensation of 1 with 4-fluoro (17) /4-(trifluoromethyl)be nzaldehyde. 1-[4-[[2-[(4-Substitutedphenyl)methylene] hydrazino]-carbonyl]phenyl]-3-substituted thioureas $\mathbf{4 a - g}$ and 5a-f were synthesized by the reaction of 4 -amino- $N^{\prime}-[(4-$ fluoro/4-(trifluoromethyl)phenyl]methylene]benzohydrazide
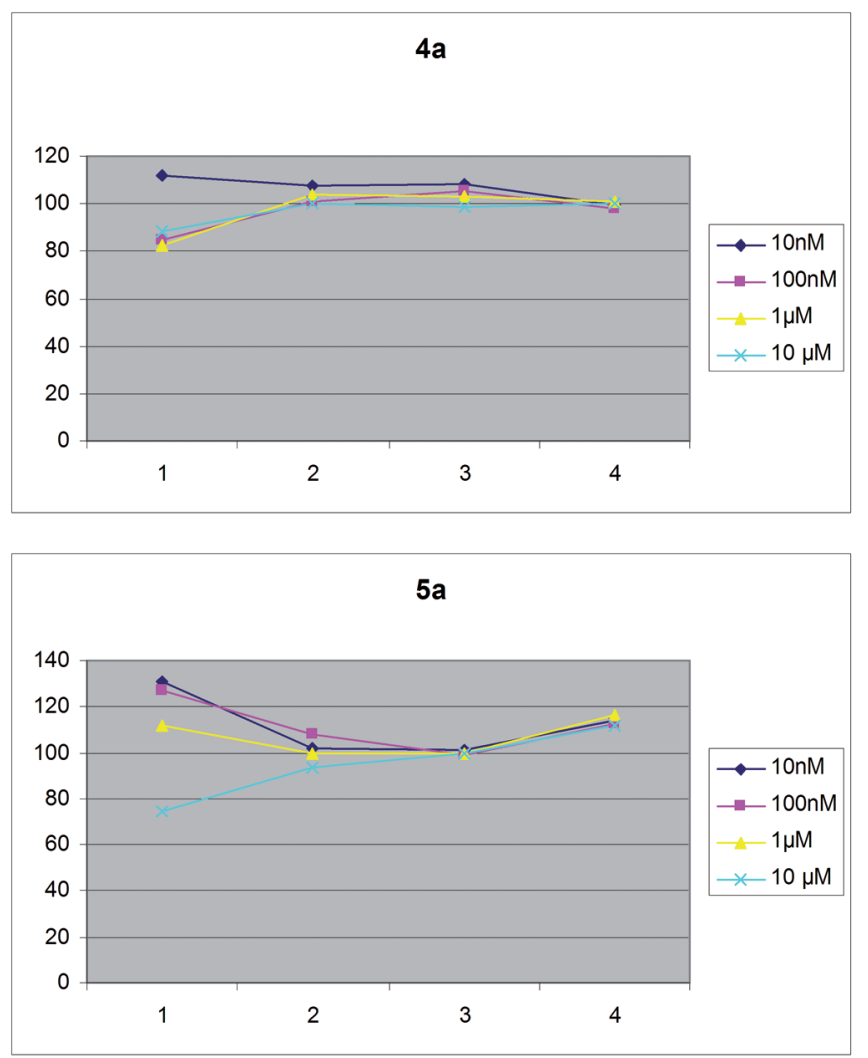

with substituted phenyl isothiocyanates in dry acetonitrile in yields between 42 and 68\% (Scheme 1). The reaction for thiourea was reported to be performed in certain dry solvents or mixtures (18-21). In the present study, dry acetonitrile was tried and found to be useful. The physical and spectral data of thioureas 4a-g and 5a-f are given in Tables 1 and 2.

4-Amino- $N^{\prime}$-[(4-fluorophenyl)methylene]benzohydrazide, which was reported to possess a weak inhibitory potency against $M$. tuberculosis H37Rv at $12.5 \mu \mathrm{g} / \mathrm{ml}(14)$, and 4-amino$N^{\prime}$-[[4-(trifluoromethyl)phenyl]methylene]benzohydrazide which was originally synthesized in the present study, were chosen as starting compounds to design several novel thioureas. The ${ }^{1} \mathrm{H}-\mathrm{NMR}$ spectra of $\mathbf{4 a - g}$ and $\mathbf{5 a}-\mathbf{f}$ showed single signals corresponding to resonances of azomethine protons at 8.38-8.51 ppm (22). In ${ }^{1} \mathrm{H}-\mathrm{NMR}$ spectra, findings such as resonances at 8.44-10.03 and 9.84-10.24 ppm due to thiourea R-NHCS- and -CS-NH-Ar function (15), respectively, and the lack of resonances attributable to $\mathrm{NH}_{2}$ function supports the formation of the expected thiourea structures. Remaining chemical shifts were also recorded at expected values.

High resolution mass spectra (HRMS) confirmed the molecular weights and empirical formula of the compounds $4 \mathbf{a}-\mathbf{g}$ and 5a-f , with less than $8 \mathrm{mmu}$ bias between calculated and experimental $\mathrm{m} / \mathrm{z}$ values of either molecular or fragment ions (Table 2). Ionization mode was electron impact (EI) in case of compound $4 \mathrm{c}$ whereas remaining compounds did not give molecular ion peaks using this technique. These compounds were analyzed using fast atomic bombardment (FAB) procedure giving exact $\mathrm{MH}^{+}$peaks instead of $\mathrm{M}^{+}$in 3-nitrobenzyl alcohol matrix. Fragmentation pattern for the representative compound $4 \mathrm{c}$ which is given in Scheme 2, also supported the expected structure. First fragmentation was cleavage of thiourea moiety yielding isothiocyanate fragment at m/z 299.0529 via benzyl loss which was detected. Characteristic fragmentations for hydrazide-hydrazones were also observed. Main fragmentation product was observed as 4-aminophenyl carbonyl cation, giving the base peak at $\mathrm{m} / \mathrm{z} 120.0444$.

\section{Antiviral activity}

Compounds $4 \mathbf{a}-\mathrm{g}$ and 5a-f were tested for antiviral activity and cytotoxicity in various viral test systems (Tables 3-5), according to previously published procedures (23-27). The following viruses and host cells were used for the evaluation :

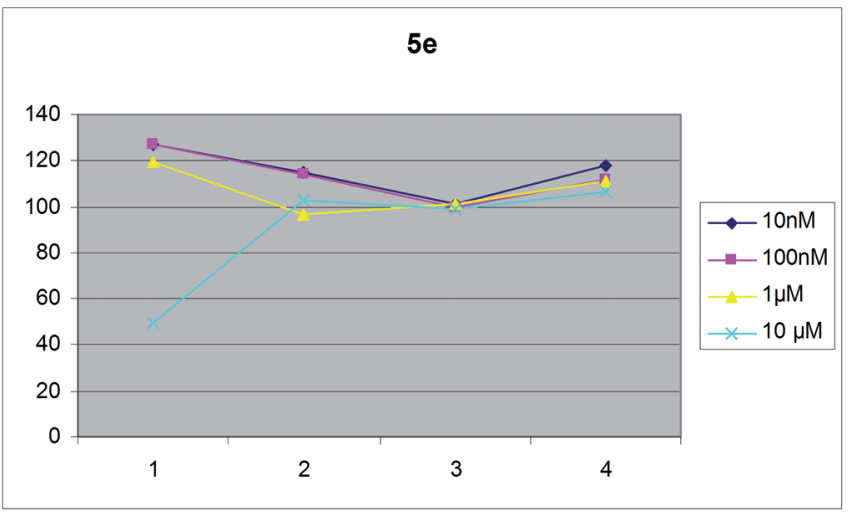

FIGURE 2. Cytotoxic effects of the compounds $4 \mathrm{a}, 5 \mathrm{a}$, 5e at four different concentrations (10 $\mathrm{nM}, 100 \mathrm{nM}, 1 \mu \mathrm{M}, 10 \mu \mathrm{M})$. 


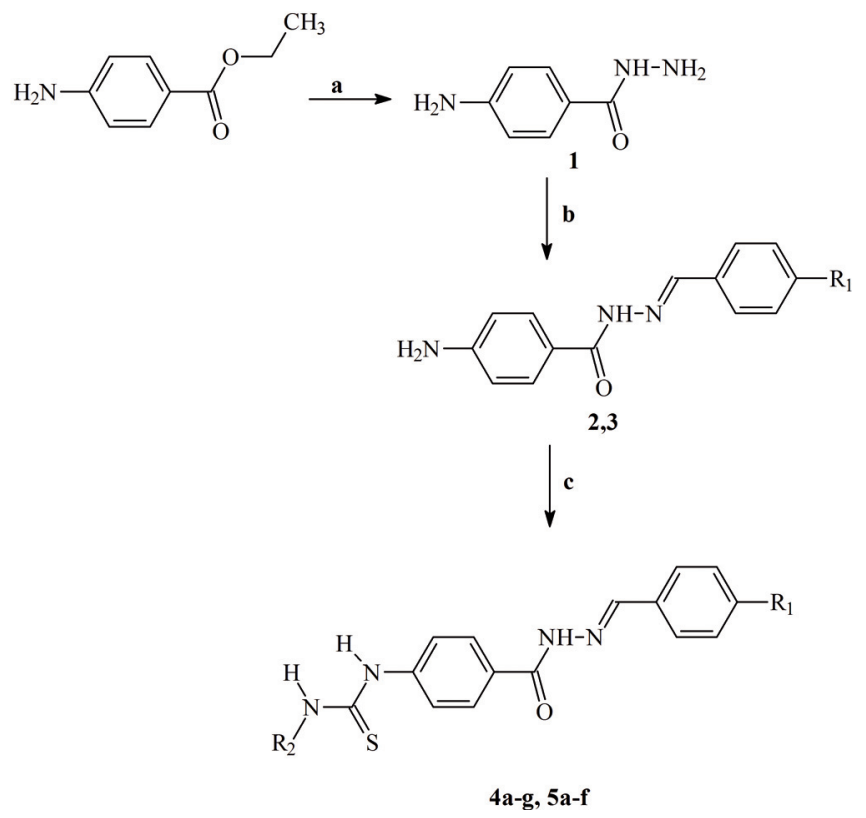

SCHEME 1. Synthetic route to compounds 2, 3, 4a-g and 5a-f. Reagents and conditions : (a) $\mathrm{H}_{2} \mathrm{~N}-\mathrm{NH}_{2} \cdot \mathrm{H}_{2} \mathrm{O} / \mathrm{EtOH}$, reflux; (b) $\mathrm{R}_{1}-\mathrm{C}_{6} \mathrm{H}_{4}-\mathrm{CH}=\mathrm{O} / \mathrm{EtOH}$, reflux ; (c) $\mathrm{R}_{2}-\mathrm{C}_{6} \mathrm{H}_{4}-\mathrm{NCS} /$ dry acetonitrile, reflux.

(a) Vero cell kultures : Parainfluenza-3 virus, Reovirus-1, Sindbis virus, Punto Toro virus and Coxsackie B4 virus.

(b) HeLa cell cultures : Vesicular stomatitis virus (VSV), Coxsackie B4 virus and respiratory syncytial virus.

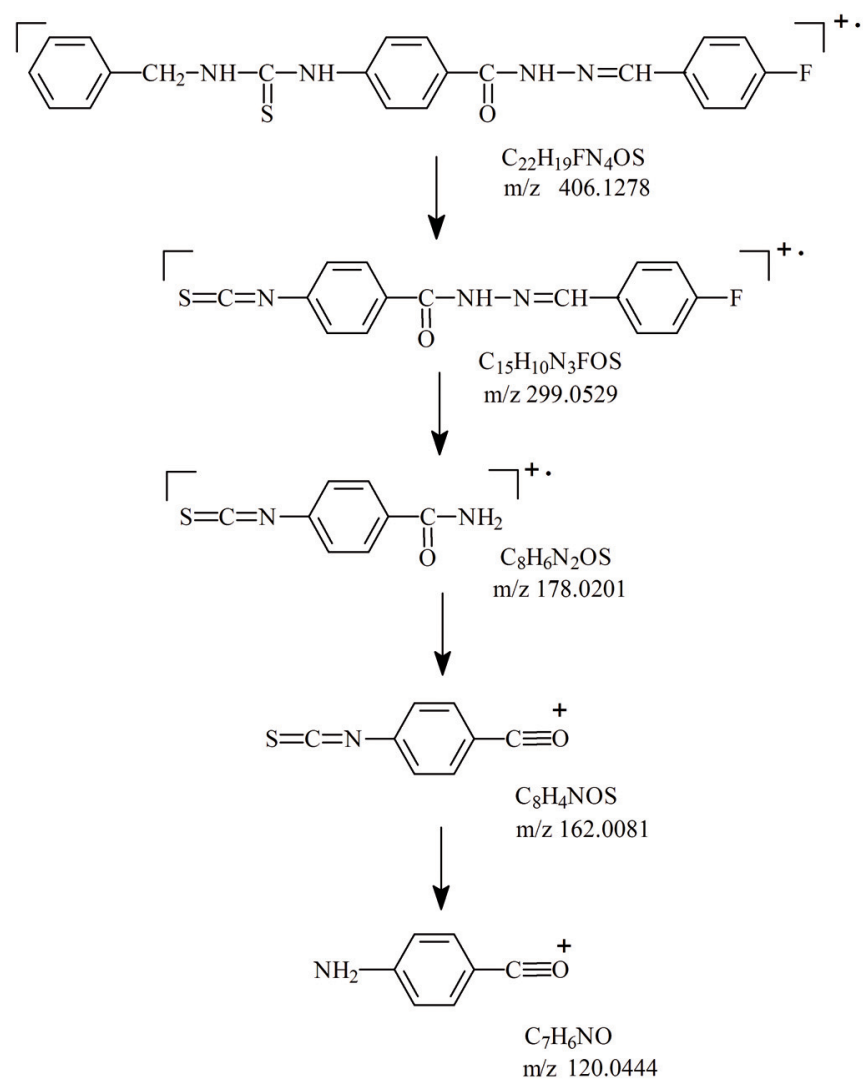

SCHEME 2. HR-El mass spectral fragmentation of 4c.

TABLE 1. Physical properties and elemental analysis data of 4a-g and 5a-f.

\begin{tabular}{|c|c|c|c|c|c|c|c|c|c|c|}
\hline \multirow[t]{2}{*}{ Compd } & \multirow[t]{2}{*}{$R_{1}$} & \multirow[t]{2}{*}{$\mathrm{R}_{2}$} & \multirow[t]{2}{*}{ Formula } & \multirow[t]{2}{*}{ M.W. } & \multirow[t]{2}{*}{$\begin{array}{l}\text { Color } \\
\text { M.p }\left({ }^{\circ} \mathrm{C}\right)\end{array}$} & \multirow[t]{2}{*}{ Yield* (\%) } & \multicolumn{4}{|c|}{$\begin{array}{l}\text { Elemental Analysis } \\
\text { (Calculated / Found) }\end{array}$} \\
\hline & & & & & & & $\mathrm{C}$ & $\mathrm{H}$ & $\mathrm{N}$ & $S$ \\
\hline $4 a$ & $-\mathrm{F}$ & $-\mathrm{C}_{6} \mathrm{H}_{5}$ & $\mathrm{C}_{21} \mathrm{H}_{17} \mathrm{FN}_{4} \mathrm{OS} .1 / 2 \mathrm{H}_{2} \mathrm{O}$ & 401.456 & $\begin{array}{l}\text { White } \\
238\end{array}$ & 42 & $\begin{array}{l}62.83 \\
62.57\end{array}$ & $\begin{array}{l}4.52 \\
4.08\end{array}$ & $\begin{array}{l}13.96 \\
14.60\end{array}$ & $\begin{array}{l}7.98 \\
8.30\end{array}$ \\
\hline $4 b$ & $-F$ & $-\mathrm{C}_{6} \mathrm{H}_{4}-\mathrm{OCH}_{3}$ & $\mathrm{C}_{22} \mathrm{H}_{19} \mathrm{FN}_{4} \mathrm{O}_{2} \mathrm{~S}$ & 422.475 & $\begin{array}{l}\text { White } \\
231-4\end{array}$ & 55 & $\begin{array}{l}62.54 \\
61.94\end{array}$ & $\begin{array}{l}4.53 \\
4.17\end{array}$ & $\begin{array}{l}13.26 \\
13.83\end{array}$ & $\begin{array}{l}7.59 \\
7.75\end{array}$ \\
\hline $4 c$ & $-F$ & $-\mathrm{CH}_{2} \mathrm{C}_{6} \mathrm{H}_{5}$ & $\mathrm{C}_{22} \mathrm{H}_{19} \mathrm{FN}_{4} \mathrm{OS}$ & 406.476 & $\begin{array}{l}\text { White } \\
242\end{array}$ & 58 & $\begin{array}{l}65.01 \\
64.28\end{array}$ & $\begin{array}{l}4.71 \\
4.52\end{array}$ & $\begin{array}{l}13.78 \\
13.81\end{array}$ & $\begin{array}{l}7.89 \\
8.77\end{array}$ \\
\hline $4 d$ & $-F$ & $-\mathrm{C}_{6} \mathrm{H}_{4}-\mathrm{Br}$ & $\mathrm{C}_{21} \mathrm{H}_{16} \mathrm{BrFN}_{4} \mathrm{OS}$ & 471.345 & $\begin{array}{l}\text { Whitish cream } \\
240\end{array}$ & 59 & $\begin{array}{l}53.51 \\
53.09\end{array}$ & $\begin{array}{l}3.42 \\
3.30\end{array}$ & $\begin{array}{l}11.89 \\
11.75\end{array}$ & $\begin{array}{l}6.80 \\
7.72\end{array}$ \\
\hline $4 \mathrm{e}$ & $-F$ & $-\mathrm{C}_{6} \mathrm{H}_{4}-\mathrm{Cl}$ & $\mathrm{C}_{21} \mathrm{H}_{16} \mathrm{ClFN}_{4} \mathrm{OS}$ & 426.894 & $\begin{array}{l}\text { White } \\
240\end{array}$ & 67 & $\begin{array}{l}59.08 \\
58.88\end{array}$ & $\begin{array}{l}3.78 \\
3.66\end{array}$ & $\begin{array}{l}13.12 \\
13.14\end{array}$ & $\begin{array}{l}7.51 \\
8.24\end{array}$ \\
\hline $4 f$ & $-F$ & $-\mathrm{C}_{6} \mathrm{H}_{4}-\mathrm{F}$ & $\mathrm{C}_{21} \mathrm{H}_{16} \mathrm{~F}_{2} \mathrm{~N}_{4} \mathrm{OS} \cdot 1 / 2 \mathrm{H}_{2} \mathrm{O}$ & 419.448 & $\begin{array}{l}\text { Whitish cream } \\
244\end{array}$ & 68 & $\begin{array}{l}60.13 \\
60.26\end{array}$ & $\begin{array}{l}4.08 \\
3.96\end{array}$ & $\begin{array}{l}13.35 \\
14.39\end{array}$ & $\begin{array}{l}7.64 \\
7.33\end{array}$ \\
\hline $4 g$ & $-F$ & $-\mathrm{C}_{6} \mathrm{H}_{4}-\mathrm{CH}_{3}$ & $\mathrm{C}_{22} \mathrm{H}_{19} \mathrm{FN}_{4} \mathrm{OS} .1 / 2 \mathrm{H}_{2} \mathrm{O}$ & 415.486 & $\begin{array}{l}\text { White } \\
235\end{array}$ & 45 & $\begin{array}{l}63.60 \\
63.99\end{array}$ & $\begin{array}{l}4.85 \\
4.50\end{array}$ & $\begin{array}{l}13.48 \\
13.94\end{array}$ & $\begin{array}{l}7.72 \\
8.11\end{array}$ \\
\hline $5 a$ & $-\mathrm{CF}_{3}$ & $-\mathrm{C}_{6} \mathrm{H}_{5}$ & $\mathrm{C}_{22} \mathrm{H}_{17} \mathrm{~F}_{3} \mathrm{~N}_{4} \mathrm{OS} .1 / 2 \mathrm{H}_{2} \mathrm{O}$ & 451.464 & $\begin{array}{l}\text { White } \\
265-8\end{array}$ & 46 & $\begin{array}{l}58.53 \\
58.50\end{array}$ & $\begin{array}{l}4.02 \\
3.65\end{array}$ & $\begin{array}{l}12.41 \\
12.56\end{array}$ & $\begin{array}{l}7.10 \\
7.21\end{array}$ \\
\hline $5 b$ & $-\mathrm{CF}_{3}$ & $\mathrm{C}_{6} \mathrm{H}_{4}-\mathrm{OCH}_{3}$ & $\mathrm{C}_{23} \mathrm{H}_{19} \mathrm{~F}_{3} \mathrm{~N}_{4} \mathrm{O}_{2} \mathrm{~S} .1 / 2 \mathrm{H}_{2} \mathrm{O}$ & 481.490 & $\begin{array}{l}\text { White } \\
250\end{array}$ & 47 & $\begin{array}{l}57.37 \\
57.43\end{array}$ & $\begin{array}{l}4,19 \\
3.63\end{array}$ & $\begin{array}{l}11.64 \\
12.28\end{array}$ & $\begin{array}{l}6.66 \\
6.03\end{array}$ \\
\hline $5 c$ & $-\mathrm{CF}_{3}$ & $-\mathrm{C}_{6} \mathrm{H}_{4}-\mathrm{Br}$ & $\mathrm{C}_{22} \mathrm{H}_{16} \mathrm{BrF}_{3} \mathrm{~N}_{4} \mathrm{OS}$ & 521.353 & $\begin{array}{l}\text { White } \\
245-8\end{array}$ & 59 & $\begin{array}{l}50.68 \\
50.72\end{array}$ & $\begin{array}{l}3.09 \\
3.13\end{array}$ & $\begin{array}{l}10.75 \\
10.86\end{array}$ & $\begin{array}{l}6.15 \\
6.63\end{array}$ \\
\hline $5 d$ & $-\mathrm{CF}_{3}$ & $-\mathrm{C}_{6} \mathrm{H}_{4}-\mathrm{Cl}$ & $\mathrm{C}_{22} \mathrm{H}_{16} \mathrm{CIF}_{3} \mathrm{~N}_{4} \mathrm{OS}$ & 476.902 & $\begin{array}{l}\text { Whitish cream } \\
247\end{array}$ & 58 & $\begin{array}{l}55.41 \\
54.96\end{array}$ & $\begin{array}{l}3.38 \\
3.36\end{array}$ & $\begin{array}{l}11.75 \\
11.71\end{array}$ & $\begin{array}{l}6.72 \\
7.27\end{array}$ \\
\hline $5 e$ & $-\mathrm{CF}_{3}$ & $\mathrm{C}_{6} \mathrm{H}_{4}-\mathrm{F}$ & $\mathrm{C}_{22} \mathrm{H}_{16} \mathrm{~F}_{4} \mathrm{~N}_{4} \mathrm{OS} .1 / 2 \mathrm{H}_{2} \mathrm{O}$ & 469.455 & $\begin{array}{l}\text { White } \\
260\end{array}$ & 57 & $\begin{array}{l}56.28 \\
55.94\end{array}$ & $\begin{array}{l}3.65 \\
3.43\end{array}$ & $\begin{array}{l}11.93 \\
12.66\end{array}$ & $\begin{array}{l}6.83 \\
6.38\end{array}$ \\
\hline $5 f$ & $-\mathrm{CF}_{3}$ & $-\mathrm{C}_{6} \mathrm{H}_{4}-\mathrm{CH}_{3}$ & $\mathrm{C}_{23} \mathrm{H}_{19} \mathrm{~F}_{3} \mathrm{~N}_{4} \mathrm{OS} .2 \mathrm{H}_{2} \mathrm{O}$ & 419.514 & $\begin{array}{l}\text { White } \\
245\end{array}$ & 54 & $\begin{array}{l}56.09 \\
56.39\end{array}$ & $\begin{array}{l}4.71 \\
3.78\end{array}$ & $\begin{array}{l}11.38 \\
12.06\end{array}$ & $\begin{array}{l}6.51 \\
6.67\end{array}$ \\
\hline
\end{tabular}

* Recrystallization solvent : dry acetonitrile. 
TABLE 2. IR, ${ }^{1} \mathrm{H}-\mathrm{NMR}$ and $H R$ mass spectral data of $4 \mathrm{a}-\mathrm{g}$ and $5 \mathrm{a}-\mathrm{f}$.

\begin{tabular}{|c|c|c|c|c|c|}
\hline \multirow[t]{2}{*}{ Compd } & \multirow{2}{*}{$\begin{array}{l}\text { IR } v\left(\mathrm{~cm}^{-1}\right) \\
\mathrm{NH}, \mathrm{C}=\mathrm{O}, \mathrm{C}=\mathrm{S}\end{array}$} & \multirow[t]{2}{*}{${ }^{1} \mathrm{H}-\mathrm{NMR}\left(\mathrm{DMSO}-\mathrm{d}_{6}, \mathrm{ppm}\right)$} & \multicolumn{3}{|c|}{ HR-MS (m/z) } \\
\hline & & & Calculated & Found & \\
\hline $4 a$ & $3321,1655,1238$ & $\begin{array}{l}\text { 7.12-7.90 (m, 13H, Ar-H); } 8.44(\mathrm{~s}, 1 \mathrm{H}, \mathrm{CH}=\mathrm{N}) ; 9.96-10.07 \text { (d, 1H, NH-CS-); } \\
10,22(\mathrm{~b}, 1 \mathrm{H}, \mathrm{NH}-\mathrm{CS}-) ; 11.78(\mathrm{~d}, 1 \mathrm{H}, \mathrm{CO}-\mathrm{NH})\end{array}$ & 393.1180 & $(\mathrm{FAB})$ & $393.1202\left(\mathrm{MH}^{+}\right)$ \\
\hline $4 b$ & $3317,3236,1650,1238$ & $\begin{array}{l}3.74\left(\mathrm{t}, 3 \mathrm{H}, \mathrm{O}-\mathrm{CH}_{3}\right) ; 6.90-7.90(\mathrm{~m}, 12 \mathrm{H}, \mathrm{Ar}-\mathrm{H}) ; 8.44(\mathrm{~s}, 1 \mathrm{H}, \mathrm{CH}=\mathrm{N}) ; 9.81,9.84 \\
(2 \mathrm{~s}, 1 \mathrm{H}, \mathrm{NH}-\mathrm{CS}-\mathrm{NH}) ; 10.21(\mathrm{~s}, 1 \mathrm{H}, \mathrm{CS}-\mathrm{NH}) ; 11.77(\mathrm{~d}, 1 \mathrm{H}, \mathrm{CO}-\mathrm{NH})\end{array}$ & 423.1286 & $(\mathrm{FAB})$ & $423.1328\left(\mathrm{MH}^{+}\right)$ \\
\hline $4 \mathrm{C}$ & $3283,1651,1234$ & $\begin{array}{l}4.75\left(\mathrm{~d}, 2 \mathrm{H}, \mathrm{N}-\mathrm{CH}_{2}\right) ; 7.24-7.87(\mathrm{~m}, 13 \mathrm{H}, \mathrm{Ar}-\mathrm{H}) ; 8.38-8.44(\mathrm{~d}, 2 \mathrm{H}, \mathrm{CH}=\mathrm{N} \text { and } \\
\mathrm{NH}-9.85(\mathrm{~s}, 1 \mathrm{H}, \mathrm{CS}-\mathrm{NH}) ; 11.76(\mathrm{~s}, 1 \mathrm{H}, \mathrm{CO}-\mathrm{NH}) .\end{array}$ & 406.1264 & $(E I)$ & $406.1278\left(\mathrm{M}^{+}\right)$ \\
\hline $4 d$ & $3294,3232,1654,1242$ & $\begin{array}{l}\text { 7.25-7.87 (m, 12H, Ar-H); } 8.43(\mathrm{~s}, 1 \mathrm{H}, \mathrm{CH}=\mathrm{N}) ; 10.01-10.07 \text { (d, 2H, NH-CS- } \\
\mathrm{NH}) ; 11.77(\mathrm{~s}, 1 \mathrm{H}, \mathrm{CO}-\mathrm{NH}) .\end{array}$ & 471.0285 & $(\mathrm{FAB})$ & $\begin{array}{l}471.0301\left(\mathrm{MH}^{+}\right) \\
473.0308\left(\mathrm{MH}^{+}+2\right)\end{array}$ \\
\hline $4 \mathrm{e}$ & $3320,3236,1659,1245$ & $\begin{array}{l}\text { 7.24-7.87 (m, 12H, Ar-H); } 8.44(\mathrm{~s}, 1 \mathrm{H}, \mathrm{CH}=\mathrm{N}) ; 10.02-10.07 \text { (d, 2H, NH-CS- } \\
\mathrm{NH}) ; 11.76(\mathrm{~s}, 1 \mathrm{H}, \mathrm{CO}-\mathrm{NH}) \text {. }\end{array}$ & 427.0790 & $(\mathrm{FAB})$ & $\begin{array}{l}427.0827\left(\mathrm{MH}^{+}\right) \\
429.0705\left(\mathrm{MH}^{+}+2\right)\end{array}$ \\
\hline $4 f$ & $3217,1643,1238$ & $\begin{array}{l}\text { 7.14-7.90 (m, 12H, Ar-H); } 8.44(\mathrm{~s}, 1 \mathrm{H}, \mathrm{CH}=\mathrm{N}) ; 9.91,10.05 \text { (2s, 1H,NH-CS-); } \\
10.21 \text { (s,1H, NH-CS-); 11.76-11.78 (d, 1H, CO-NH). }\end{array}$ & 411.1086 & $(\mathrm{FAB})$ & $411.1108\left(\mathrm{MH}^{+}\right)$ \\
\hline $4 \mathrm{~g}$ & $3321,3236,1655,1238$ & $\begin{array}{l}2.27\left(\mathrm{~s}, 3 \mathrm{H}, \mathrm{C}_{6} \mathrm{H}_{4} \mathrm{CH}_{3}\right) ; 7.13-7.90(\mathrm{~m}, 12 \mathrm{H}, \mathrm{Ar}-\mathrm{H}) ; 8.44(\mathrm{~s}, 1 \mathrm{H}, \mathrm{CH}=\mathrm{N}) ; 9.87- \\
9.91(\mathrm{~d} 1 \mathrm{H}, \mathrm{NH}-\mathrm{CS}) ; 10.21(\mathrm{~b}, 1 \mathrm{H}, \mathrm{NH}-\mathrm{CS}) ; 11.76-11.78(\mathrm{~d}, 1 \mathrm{H}, \mathrm{CO}-\mathrm{NH}) .\end{array}$ & 407.1336 & $(\mathrm{FAB})$ & $407.1324\left(\mathrm{MH}^{+}\right)$ \\
\hline $5 a$ & $3321,3236,1655,1238$ & $\begin{array}{l}\text { 7.12-7.94 (m, 13H, Ar-H); } 8.51(\mathrm{~s}, 1 \mathrm{H}, \mathrm{CH}=\mathrm{N}) ; 9.97-10.02 \text { (d, } 1 \mathrm{H}, \mathrm{NH}-\mathrm{CS}) \\
10.24 \text { (b } 1 \mathrm{H}, \mathrm{NH}-\mathrm{CS}-) ; 11.95(\mathrm{~s}, 1 \mathrm{H}, \mathrm{CO}-\mathrm{NH})\end{array}$ & 443.1148 & $(\mathrm{FAB})$ & $443.1132\left(\mathrm{MH}^{+}\right)$ \\
\hline $5 b$ & $3301,3240,1651,1249$ & $\begin{array}{l}3.83\left(\mathrm{t}, 3 \mathrm{H}, \mathrm{O}-\mathrm{CH}_{3}\right) ; 6.90-7.92(\mathrm{~m}, 12 \mathrm{H}, \mathrm{Ar}-\mathrm{H}) ; 8.51(\mathrm{~s}, 1 \mathrm{H}, \mathrm{CH}=\mathrm{N}) ; 9.79-9.86 \\
(\mathrm{~d}, 1 \mathrm{H}, \mathrm{NH}-\mathrm{CS}) ; 10.24(\mathrm{~s}, 1 \mathrm{H}, \mathrm{CS}-\mathrm{NH}) ; 11.96(\mathrm{~d}, 1 \mathrm{H}, \mathrm{CO}-\mathrm{NH}) .\end{array}$ & 473.1254 & $(\mathrm{FAB})$ & $473.1258\left(\mathrm{MH}^{+}\right)$ \\
\hline $5 c$ & $3309,3232,1655,1257$ & $\begin{array}{l}7.42-7.94(\mathrm{~m}, 12 \mathrm{H}, \mathrm{Ar}-\mathrm{H}) ; 8.51(\mathrm{~s}, 1 \mathrm{H}, \mathrm{CH}=\mathrm{N}) ; 10.03(\mathrm{~s}, 1 \mathrm{H}, \mathrm{NH}-\mathrm{CS}) ; 10.10(\mathrm{~s}, \\
1 \mathrm{H}, \mathrm{CS}-\mathrm{NH}) ; 11.95(\mathrm{~s}, 1 \mathrm{H}, \mathrm{CO}-\mathrm{NH}) .\end{array}$ & 521.0253 & $(\mathrm{FAB})$ & $\begin{array}{l}521.0250\left(\mathrm{MH}^{+}\right) \\
523.0242\left(\mathrm{MH}^{+}+2\right)\end{array}$ \\
\hline $5 d$ & $3309,1655,1172$ & $\begin{array}{l}\text { 7.38-7.94 (m, 12H, Ar-H); } 8.51(\mathrm{~s}, 1 \mathrm{H}, \mathrm{CH}=\mathrm{N}) ; 10.03-10.09(\mathrm{~d}, 2 \mathrm{H}, \mathrm{NH}-\mathrm{CS}- \\
\mathrm{NH}) ; 11.96(\mathrm{~s}, 1 \mathrm{H}, \mathrm{CO}-\mathrm{NH}) .\end{array}$ & 477.0758 & $(\mathrm{FAB})$ & $\begin{array}{l}477.0743\left(\mathrm{MH}^{+}\right) \\
479.0733\left(\mathrm{MH}^{+}+2\right)\end{array}$ \\
\hline $5 e$ & $3317,3202,1651,1172$ & $\begin{array}{l}\text { 7.15-7.92 (m, 12H, Ar-H); } 8.51(\mathrm{~s}, 1 \mathrm{H}, \mathrm{CH}=\mathrm{N}) ; 9.92-10.02(2 \mathrm{~s}, 1 \mathrm{H}, \mathrm{NH}-\mathrm{CS}) \\
\text { 10.24 (s, } 1 \mathrm{H}, \mathrm{CS}-\mathrm{NH}) ; 11.97(\mathrm{~s}, 1 \mathrm{H}, \mathrm{CO}-\mathrm{NH})\end{array}$ & 461.1054 & $(\mathrm{FAB})$ & $461.1049\left(\mathrm{MH}^{+}\right)$ \\
\hline $5 f$ & $3317,3236,1651,1165$ & $\begin{array}{l}2.32\left(\mathrm{~s}, 3 \mathrm{H}, \mathrm{C}_{6} \mathrm{H}_{4} \mathrm{CH}_{3}\right) ; 7.13-7.92(\mathrm{~m}, 12 \mathrm{H}, \mathrm{Ar}-\mathrm{H}) ; 8.51(\mathrm{~s}, 1 \mathrm{H}, \mathrm{CH}=\mathrm{N}) ; 9.91- \\
10.24(\mathrm{~d}, \mathrm{~b}, 2 \mathrm{H}, \mathrm{NH}-\mathrm{CS}-\mathrm{NH}) ; 11.95(\mathrm{~s}, 1 \mathrm{H}, \mathrm{CO}-\mathrm{NH}) .\end{array}$ & 457.1304 & $(\mathrm{FAB})$ & $457.1335\left(\mathrm{MH}^{+}\right)$ \\
\hline
\end{tabular}

TABLE 3. Cytotoxicity and antiviral activity of compounds in Hel, HeLa and Vero cell cultures

\begin{tabular}{|c|c|c|c|c|c|c|c|c|c|c|c|c|c|c|c|c|}
\hline \multirow[t]{3}{*}{ Compound } & \multicolumn{6}{|c|}{ HEL cell cultures } & \multicolumn{4}{|c|}{ HELA cell cultures } & \multicolumn{6}{|c|}{ Vero cell cultures } \\
\hline & \multirow{2}{*}{$\begin{array}{c}\text { Min. } \\
\text { cytotoxic } \\
\text { conc. }^{\text {a }} \\
(\mu \mathrm{g} / \mathrm{ml})\end{array}$} & \multicolumn{5}{|c|}{ Min. inhibitory conc. ${ }^{b}(\mu \mathrm{g} / \mathrm{ml})$} & \multirow{2}{*}{$\begin{array}{c}\text { Min. } \\
\text { cytotoxic } \\
\text { conc. }{ }^{a} \\
(\mu \mathrm{g} / \mathrm{ml})\end{array}$} & \multicolumn{3}{|c|}{ Min. inhibitory conc. ${ }^{b}(\mu \mathrm{g} / \mathrm{ml})$} & \multirow{2}{*}{$\begin{array}{c}\text { Min. } \\
\text { cytotoxic } \\
\text { conc. }^{a} \\
(\mu \mathrm{g} / \mathrm{ml})\end{array}$} & \multicolumn{5}{|c|}{ Min. inhibitory conc. ${ }^{b}(\mu \mathrm{g} / \mathrm{ml})$} \\
\hline & & $\begin{array}{l}\text { Herpes } \\
\text { simplex } \\
\text { virus-1 } \\
\text { (KOS) }\end{array}$ & $\begin{array}{c}\text { Herpes } \\
\text { simplex } \\
\text { virus-2 } \\
\text { (G) }\end{array}$ & $\begin{array}{c}\text { Vaccinia } \\
\text { virus }\end{array}$ & $\begin{array}{c}\text { Vesicular } \\
\text { stomatitis } \\
\text { virus }\end{array}$ & $\begin{array}{c}\text { Herpes } \\
\text { simplex } \\
\text { virus-1 } \\
\text { TK KOS } \\
\text { ACVr }\end{array}$ & & $\begin{array}{l}\text { Vesicular } \\
\text { stomatitis } \\
\text { virus }\end{array}$ & $\begin{array}{c}\text { Coxsackie } \\
\text { B4 virus }\end{array}$ & $\begin{array}{c}\text { Respiratory } \\
\text { syncytial } \\
\text { virus }\end{array}$ & & $\begin{array}{c}\text { Para } \\
\text { I influenza-3 } \\
\text { virus }\end{array}$ & $\begin{array}{c}\text { Reo } \\
3 \text { virus- } \\
1\end{array}$ & $\begin{array}{c}\text { Sindbis } \\
\text { virus }\end{array}$ & $\begin{array}{c}\text { Coxsackie } \\
\text { B4 virus }\end{array}$ & $\begin{array}{c}\text { Punta } \\
\text { Toro } \\
\text { virus }\end{array}$ \\
\hline $4 a$ & 8 & $>1.6$ & $>1.6$ & $>1.6$ & $>1.6$ & $>1.6$ & 40 & $>8$ & $>8$ & $>8$ & 40 & $>8$ & $>8$ & $>8$ & $>8$ & $>8$ \\
\hline $4 b$ & 8 & $>1.6$ & $>1.6$ & $>1.6$ & $>1.6$ & $>1.6$ & 40 & $>8$ & $>8$ & $>8$ & 40 & $>8$ & $>8$ & $>8$ & $>8$ & $>8$ \\
\hline $4 c$ & 8 & $>1.6$ & $>1.6$ & $>1.6$ & $>1.6$ & $>1.6$ & 8 & $>1.6$ & $>1.6$ & $>1.6$ & 8 & $>1.6$ & $>1.6$ & $>1.6$ & $>1.6$ & $>1.6$ \\
\hline $4 d$ & 8 & $>1.6$ & $>1.6$ & $>1.6$ & $>1.6$ & $>1.6$ & 200 & $>40$ & $>40$ & $>40$ & 40 & $>8$ & $>8$ & $>8$ & $>8$ & $>8$ \\
\hline $4 \mathrm{e}$ & 8 & $>1.6$ & $>1.6$ & $>1.6$ & $>1.6$ & $>1.6$ & 40 & $>8$ & $>8$ & $>8$ & 40 & $>8$ & $>8$ & $>8$ & $>8$ & $>8$ \\
\hline $4 f$ & 8 & $>1.6$ & $>1.6$ & $>1.6$ & $>1.6$ & $>1.6$ & 40 & $>8$ & $>8$ & $>8$ & 40 & $>8$ & $>8$ & $>8$ & $>8$ & $>8$ \\
\hline $4 g$ & 8 & $>1.6$ & $>1.6$ & $>1.6$ & $>1.6$ & $>1.6$ & 40 & $>8$ & $>8$ & $>8$ & 40 & $>8$ & $>8$ & $>8$ & $>8$ & $>8$ \\
\hline $5 a$ & 8 & $>1.6$ & $>1.6$ & $>1.6$ & $>1.6$ & $>1.6$ & 40 & $>8$ & $>8$ & $>8$ & 40 & $>8$ & $>8$ & $>8$ & $>8$ & $>8$ \\
\hline $5 b$ & 8 & $>1.6$ & $>1.6$ & $>1.6$ & $>1.6$ & $>1.6$ & 200 & $>40$ & $>40$ & $>40$ & 40 & $>8$ & $>8$ & $>8$ & $>8$ & $>8$ \\
\hline $5 c$ & 8 & $>1.6$ & $>1.6$ & $>1.6$ & $>1.6$ & $>1.6$ & 200 & $>40$ & $>40$ & $>40$ & 40 & $>8$ & $>8$ & $>8$ & $>8$ & $>8$ \\
\hline $5 d$ & 8 & $>1.6$ & $>1.6$ & $>1.6$ & $>1.6$ & $>1.6$ & 40 & $>8$ & $>8$ & $>8$ & 40 & $>8$ & $>8$ & $>8$ & $>8$ & $>8$ \\
\hline $5 e$ & 8 & $>1.6$ & $>1.6$ & $>1.6$ & $>1.6$ & $>1.6$ & 200 & $>40$ & $>40$ & $>40$ & 40 & $>8$ & $>8$ & $>8$ & $>8$ & $>8$ \\
\hline $5 f$ & 8 & $>1.6$ & $>1.6$ & $>1.6$ & $>1.6$ & $>1.6$ & 200 & $>40$ & $>40$ & $>40$ & 40 & $>8$ & $>8$ & $>8$ & $>8$ & $>8$ \\
\hline $\begin{array}{l}\text { Brivudin } \\
(\mu \mathrm{M})\end{array}$ & $>250$ & 0.08 & 10 & 2 & $>250$ & $>250$ & $>250$ & $>250$ & $>250$ & $>250$ & $>250$ & $>250$ & $>250$ & $>250$ & $>250$ & $>250$ \\
\hline $\begin{array}{l}\text { Ribavirin } \\
(\mu \mathrm{M})\end{array}$ & $>250$ & 250 & 250 & 150 & 150 & $>250$ & $>250$ & 30 & 150 & 50 & $>250$ & 150 & 150 & $>250$ & $>250$ & 250 \\
\hline $\begin{array}{l}\text { Acyclovir } \\
(\mu \mathrm{M})\end{array}$ & $>250$ & 0.4 & 0.4 & $>250$ & $>250$ & 50 & & & & & & & & & & \\
\hline $\begin{array}{l}\text { Ganciclovir } \\
(\mu \mathrm{M})\end{array}$ & $>100$ & 0.032 & 0.0064 & 100 & $>100$ & 2.4 & & & & & & & & & & \\
\hline $\begin{array}{l}\text { (S)-DHPA } \\
(\mu \mathrm{M})\end{array}$ & & & & & & & $>250$ & 150 & 150 & $>250$ & $>250$ & 50 & 250 & $>250$ & $>250$ & $>250$ \\
\hline
\end{tabular}


TABLE 4. Cytoxicity and antiviral activity of compounds $4 \mathrm{a}-\mathrm{g}$ and $5 \mathrm{a}-\mathrm{f}$ against cytomegalovirus (CMV) and varicella-zoster virus (VZV) in human embryonic lung (HEL) cells.

\begin{tabular}{|c|c|c|c|c|c|c|c|}
\hline \multirow[t]{3}{*}{ Compd. } & \multicolumn{4}{|c|}{ Antiviral activity $\mathrm{EC}_{50}(\mu \mathrm{g} / \mathrm{ml})^{a}$} & \multicolumn{3}{|c|}{ Cytotoxicity $(\mu \mathrm{g} / \mathrm{ml})$} \\
\hline & \multicolumn{2}{|c|}{ CMV (cytomegalovirus) } & \multicolumn{2}{|c|}{ VZV (varicella-zoster virus) } & \multicolumn{2}{|c|}{ Cell morphology $\mathrm{MCC}^{\mathrm{b}}$} & \multirow{2}{*}{$\begin{array}{l}\text { Cell growth } \\
\mathrm{CC}_{50}{ }^{\mathrm{C}}\end{array}$} \\
\hline & AD-169 strain & Davis strain & TK+ (OKA strain) & TK- (07/1 strain) & CMV assay & VZV assay & \\
\hline $4 a$ & $>4$ & $>4$ & $>4$ & $>4$ & 20 & 20 & 10.5 \\
\hline $4 b$ & $>20$ & $>20$ & $>20$ & $>4$ & 100 & $\geq 20$ & $>100$ \\
\hline $4 c$ & $>4$ & $>4$ & $>4$ & $>4$ & 20 & 20 & 12.6 \\
\hline $4 d$ & $>20$ & $>4$ & $>4$ & $>20$ & $\geq 20$ & $\geq 20$ & $>100$ \\
\hline $4 e$ & $>20$ & $>20$ & $>20$ & $>20$ & 100 & $\geq 20$ & $>100$ \\
\hline $4 f$ & $>20$ & $>100$ & $>20$ & $>20$ & $\geq 100$ & 100 & $>100$ \\
\hline $4 g$ & $>20$ & $>20$ & $>20$ & $>20$ & 100 & 100 & $>100$ \\
\hline $5 a$ & $>20$ & $>4$ & $>4$ & $>20$ & $\geq 20$ & $\geq 20$ & $>100$ \\
\hline $5 b$ & $>20$ & $>20$ & $>0.8$ & $>4$ & 100 & $\geq 0.8$ & $>100$ \\
\hline $5 c$ & $>4$ & $>4$ & $>4$ & $>4$ & 20 & $\geq 4$ & 62.2 \\
\hline $5 d$ & $>4$ & $>20$ & $>4$ & $>4$ & $\geq 20$ & 20 & $>100$ \\
\hline $5 e$ & $>20$ & $>20$ & $>4$ & $>4$ & 100 & 20 & $>100$ \\
\hline $5 f$ & $>20$ & $>4$ & $>4$ & $>4$ & $\geq 20$ & $\geq 4$ & $>100$ \\
\hline Ganciclovir & 1.4 & 1.7 & - & - & 400 & - & 80 \\
\hline Cidofovir & 0.24 & 0.37 & - & - & 400 & - & 57 \\
\hline Acyclovir & - & - & 1.0 & 15 & - & $>50$ & 190 \\
\hline Brivudin & - & - & 0.0095 & 12.6 & - & $>50$ & 244 \\
\hline $\begin{array}{ll}\text { a } & \text { Effective } \\
\text { b } & \text { Minimum } \\
\text { c } & \text { Cytotoxic }\end{array}$ & $\begin{array}{l}\text { on required to } \\
\text { oncentration th } \\
\text { ion required to }\end{array}$ & $\begin{array}{l}\text { virus-induce } \\
\text { ses a microsc } \\
\text { cell growth }\end{array}$ & $\begin{array}{l}\text { allic effect by } 5 \\
\text { ally detectable alte } \\
\% \text {. }\end{array}$ & $\begin{array}{l}\text { Virus input was } \\
\text { tion of cell morph }\end{array}$ & $\mathrm{ZV})$ or $100(\mathrm{Cl}$ & que forming & \\
\hline
\end{tabular}

(c) HEL cell culture : Herpes simplex virus type 1 (HSV-1) (KOS strain), Herpes simplex virus type 2 (HSV-2) (G strain), Vaccinia virus, Vesicular stomatitis virus , HSV-1 thymidine kinase deficient virus (TK- KOS ACV ${ }^{\mathrm{r}}$ ).

(d) HEL cell culture : Cytomegalovirus (CMV) (strains AD-169 and Davis), Varicella-zoster virus (VZV) $\left(\mathrm{TK}^{+} \mathrm{VZV}\right.$ strain OKA strain and 07/1 strain).

(e) MT-4 cells : HIV-1 (IIIB) and HIV-2 (ROD) strains.

Brivudin, (S)-DHPA, ribavirin, acyclovir, cidofovir and ganciclovir were used as the reference compounds. In the tests with viruses decribed in (a), (b), (c), (d) and (e) antiviral activity and cytotoxicity were determined with the compounds $4 \mathrm{a}-\mathrm{g}$ and 5a-f. None of synthesized compounds had selective activity at subtoxic concentrations against the viruses tested.

\section{Antitubercular activity}

Compounds 4a-g and 5a-f were also tested for in vitro antitubercular activity against M. tuberculosis H37Rv (ATCC 27294) using the BACTEC 12B medium and a broth microdilution assay, the Microplate Alamar Blue Assay (MABA) $(28,29)$. Rifampicin was used as the standard in the antitubercular assays. None of the tested compounds were considered for further antitubercular evaluation as they exhibited less than $90 \%$ inhibition in the primary screen (MIC> $>25 \mu \mathrm{g} / \mathrm{mL}$ ).

\section{Anticancer activity}

Both cytotoxicity and anticancer assay results showed that, none of the tested concentrations of the compounds gave $\mathrm{IC}_{50}$ values. Therefore, it was concluded that there were no significant differences found between cytotoxic and anticancer effects of the compounds at four different concentrations (10 $\mathrm{nM}, 100 \mathrm{nM}, 1 \mu \mathrm{M}, 10 \mu \mathrm{M})$ tested. Compounds $\mathbf{4 a}, \mathbf{5 a}$, and $\mathbf{5 e}$ caused $10-20 \%$ cytotoxic effect at the highest concentration on $4^{\text {th }}$ day of the incubation period (30) (Figure 2)

\section{EXPERIMENTAL \\ Chemistry}

All chemical compounds were purchased from Fluka. Melting points were taken on Buchi-530 apparatus. Merck silica gel 60 F254 plates were used for analytical TLC and visualized with UV. The IR spectra were obtained with a Shimadzu FTIR$8400 .{ }^{1} \mathrm{H}$ NMR spectra in DMSO- $d_{6}$ were obtained on a Bruker Avance-DPX 400 instrument. HR-Mass spectra using EI and FAB ionization techniques, were performed using a Jeol JMS700 instrument.

\section{Synthesis of 4-Aminobenzoic acid hydrazide 1 (17)}

Ethyl 4-aminobenzoate $(0.01 \mathrm{~mol})$ was added to hydrazinehydrate $(99 \%, 3 \mathrm{~mL})$. The reaction mixture was heated for $1 \mathrm{~h}$ and this reaction mixture was refluxed in the presence of ethanol. The compound thus obtained was allowed to stand overnight. The precipitated solid was washed with water, dried and cleaned twice using hot methanol.

\section{4-Amino-N'-[(4-fluoro/4-(trifluoromethyl)phenyl)methylene] benzohydrazide 2 (17), 3 \\ General procedure}

A solution of $0.01 \mathrm{~mol}$ of $\mathbf{1}$ and equimolar amount of appropriate aldehyde in $60 \mathrm{~mL}$ of ethanol was heated under reflux for 1 h (15 min for compound 3). The precipitate obtained was filtered off, washed with water and cleaned twice with boiling $\mathrm{EtOH}$.

For compound 3 yield: $47 \%$, m.p.: $225^{\circ} \mathrm{C}$ (ethanol); IR (KBr) : $\left[\mathrm{cm}^{-1}\right]$ : 3440, $3332\left(\mathrm{Ar}-\mathrm{NH}_{2}\right), 3271,3217(\mathrm{NH}), 1632(\mathrm{C}=\mathrm{O}$, hydrazone) ${ }^{1} \mathrm{H}-\mathrm{NMR} \delta$ [ppm] DMSO-d 6 : $=5.83\left(\mathrm{~s}, 2 \mathrm{H}, \mathrm{Ar}-\mathrm{NH}_{2}\right), 6.60$ 
TABLE 5. Cytoxicity and antiviral activity of compounds $4 \mathrm{a}-\mathrm{g}$ and 5a-f against HIV-I (IIIB) and HIV-II (ROD).

\begin{tabular}{|c|c|c|c|c|}
\hline \multirow[t]{2}{*}{ Compounds } & \multicolumn{2}{|c|}{ HIV-I (III $)$} & \multicolumn{2}{|c|}{ HIV-II (ROD) } \\
\hline & $\begin{array}{c}\mathrm{EC}_{50} \\
(\mu \mathrm{g} / \mathrm{ml})^{a}\end{array}$ & $\begin{array}{c}\mathrm{CC}_{50} \\
(\mu \mathrm{g} / \mathrm{ml})^{\mathrm{b}}\end{array}$ & $\begin{array}{c}\mathrm{EC}_{50} \\
(\mu \mathrm{g} / \mathrm{ml})^{\mathrm{a}}\end{array}$ & $\begin{array}{c}\mathrm{CC}_{50} \\
(\mu \mathrm{g} / \mathrm{ml})^{\mathrm{b}}\end{array}$ \\
\hline $4 a$ & $>53.85$ & 53.85 & $>53.85$ & 53.85 \\
\hline $4 b$ & $>125$ & $>125$ & $>125$ & $>125$ \\
\hline $4 c$ & $>20.3$ & 94.00 & $>16.8$ & 94.00 \\
\hline $4 d$ & $>125.00$ & $>125.00$ & $>125.00$ & $>125.00$ \\
\hline $4 \mathrm{e}$ & $>125.00$ & $>125.00$ & $>125.00$ & $>125.00$ \\
\hline $4 f$ & $>59.10$ & 59.10 & $>59.10$ & 59.10 \\
\hline $4 g$ & $>125.00$ & $>125.00$ & $>125.00$ & $>125.00$ \\
\hline $5 a$ & $>70.9$ & $>125$ & $>79.2$ & $>125$ \\
\hline $5 b$ & $>125.00$ & $>125.00$ & 75.25 & $>125.00$ \\
\hline $5 c$ & $>58.10$ & $>58.10$ & $>58.10$ & $>58.10$ \\
\hline $5 d$ & $>68.10$ & 68.10 & $>68.10$ & 68.10 \\
\hline $5 e$ & $>75.70$ & 75.70 & $>75.70$ & 75.70 \\
\hline $5 f$ & $>118.00$ & $>125.00$ & $>125.00$ & $>125.00$ \\
\hline \multicolumn{5}{|c|}{$\begin{array}{l}\text { a Effective concentration required to protect } 50 \% \text { of the cells against } \\
\text { destruction by the virus. } \\
\text { b Cytotoxic concentration required to destroy } 50 \% \text { of the uninfected host } \\
\text { cells. }\end{array}$} \\
\hline
\end{tabular}

$\left(\mathrm{d}, 2 \mathrm{H}, \mathrm{o}-\mathrm{NH}_{2}, \mathrm{~J}=8.6 \mathrm{~Hz}\right), 7.69\left(\mathrm{~d}, 2 \mathrm{H}, \mathrm{m}-\mathrm{NH}_{2}, \mathrm{~J}=8.6 \mathrm{~Hz}\right), 7.80(\mathrm{~d}$, $2 \mathrm{H}, \mathrm{o}-\mathrm{CH}, \mathrm{J}=8.3 \mathrm{~Hz}), 7.91$ (d, 2H, m-NH, J=8.3 Hz), 8.46 (d,1H, $\mathrm{CH}=\mathrm{N}), 11.65(\mathrm{~s}, 1 \mathrm{H},-\mathrm{CON} \underline{\mathrm{HN}}=\mathrm{CH}-)$; HR-MS (EI, $70 \mathrm{eV}): \mathrm{m} / \mathrm{z}$ (calculated/found) for $\mathrm{C}_{15} \mathrm{H}_{12} \mathrm{~F}_{3} \mathrm{~N}_{3} \mathrm{O} 307.0932\left[\mathrm{M}^{+}\right]$, 307.0903.

\section{1-[4-[[2-[(4-substituted phenyl)methylene] hydrazino]carb onyl]phenyl]-3-substituted thioureas 4a-g, 5a-f \\ General procedure}

A dry acetonitrile solution of 4-amino- $N^{\prime}$-[(4-fluoro/4-trifluoro phenyl)methylene]-benzohydrazide and equimolar substituted phenyl isothiocyanates in dry acetonitrile was heated under reflux for 9-15 h. The completion of reaction was checked by TLC (petroleum ether : acetone, 50:50, v/v). The precipitate obtained was filtered off and recrystallized twice with dry acetonitrile.

\section{Biological activity}

\section{Antiviral activity}

Compounds 4a-g and 5a-f were tested for antiviral activity and cytotoxicity in various viral test systems, according to previously published procedures (23-27). The synthesized compounds were tested against HIV-1 (IIIB) and HIV-2 (ROD), vesicular stomatitis virus, Coxsackie B4 virus, respiratory syncytial virus, parainfluenza-3 virus, reovirus, Sindbis virus, Punto Toro virus, herpes simplex virus type 1 and 2 and vaccinia virus-induced cytopathogenicity at subtoxic concentrations in MT-4 cells, HeLa, Vero or Hel cell culture. Brivudin, (S)-DHPA, ribavirin, acyclovir, cidofovir and ganciclovir were used as the reference compounds.

\section{Antitubercular activity}

Antitubercular evaluation was carried out in the Tuberculosis Antimicrobial Acquisition and Coordinating Facility (TAACF). Primary screen was conducted at $6.25 \mu \mathrm{g} / \mathrm{ml}$ against M.tuberculosis H37Rv in BACTEC 12B medium using both BACTEC 460 radiometric system and Microplate Alamar Blue Assay
(MABA) $(28,29)$. Compounds effecting < $90 \%$ inhibition in the primary screen (MIC $>6.25 \mathrm{~g} / \mathrm{ml}$ ) were not further evaluated. Compounds demonstrating at least $90 \%$ inhibition in the primary screen were considered for re-testing at lower concentration (MIC) in a broth microdilution MABA.

\section{Anticancer activity}

The synthesized compounds were tested for anticancer activity and cytotoxicity. The CellTiter 96 Aqueous ONE Solution (Promega, Madison, WI) was used to evaluate cellular viability utilizing reduction of 3-(4,5-dimethylthiazol-2-yl)-5-(3-carboxymethoxyphenyl)-2-(4-sulfophenyl)-2H-tetrazolium (MTS).

\section{Cell culture and viability assay}

A 549 and L 929 cell lines were used to test both anticancer effects and cytotoxicity. Cells were routinely grown in a 75-mm flask in an environment containing $5 \% \mathrm{CO}_{2}$ and passed every 3 days. Cell viability was analyzed using the MTS assay. Cells were routinely grown in a 75-mm flask in an environment containing $5 \% \mathrm{CO}_{2}$ and passed every 3 days. Cell viability was analyzed using the MTS assay. 5,000 Cells were plated in each well of a 96-well tissue culture plate. After 24 hours of growth the medium was replaced with fresh medium containing different concentration $(10 \mathrm{nM}, 100 \mathrm{nM}, 1 \mu \mathrm{M}$ and $10 \mu \mathrm{M})$ of chemicals, and the cells were grown for 4 days (30).

The MTS assay was performed according to the protocol provided by the Manufacturer. In short, $20 \mu \mathrm{L}$ of MTS solution was added to each well, and cells were incubated at $37^{\circ} \mathrm{C}$ for 1 to $3 \mathrm{~h}$. The absorbance (at $490 \mathrm{~nm}$ ) of each well was then determined. Data are presented as a percentage of the values obtained from cells cultured under the same conditions in the absence of chemicals. For the time course study of the chemicals' cytotoxicity, L 929 cells were treated with chemicals with the same dose which was used to detect anticancer effect. Cell viability was analyzed for 1-4 days after the initiation of treatment, using the MTS assay.

All test compounds were dissolved in DMSO and the final concentration of DMSO was $0.1 \%$. It was observed that the solvent showed no activity in these assays at the level that were used for screening. For comparison of the anticancer activity and cytotoxicity tests observed with the test compounds, doxorubicin and taxol were selected as standard drugs.

\section{ACKNOWLEDGEMENT}

The authors are grateful to Dr. Jürgen Gross from the Institute of Organic Chemistry, University of Heidelberg, for his generous help on obtaining HR-EI/FAB mass spectra of the synthesized compounds. We also thank Dr. Joseph A. Maddry from the Tuberculosis Antimicrobial Acquisition and Coordinating Facility (TAACF), National Institute of Allergy and Infections Diseases Southern Research Institute, GWL Hansen's Disease Center, Colorado State University, Birmingham, AL, USA, for the in vitro evaluation of antitubercular activity using M. tuberculosis H37Rv. This work was supported by the Research Fund of Marmara University, project number : SAG. YYP.290506-0097 and the GOA nr. 05/19 of the KULeuven. We thank L. Persoons, L. Van den Heurck, K. Erven, Steven Carmans, and Anita Camps for excellent technical assistance with (some of) the antiviral activity assays. 


\section{4-Aminobenzohidrazit hidrazonlarından türetilmiş bazı yeni tiyoürelerin sentezi, antiviral, antitü-} berküler ve antikanser etkilerinin değerlendirilmesi

ÖZET: Bazı yeni 1-[4-[[2-[(4-sübstitüe fenil)metilen]hidrazino]karbonil]fenil]-3-sübstitüe tiyoüre türevleri, 4-aminobenzoik asit hidraziti ile 4-fluorobenzaldehit veya 4-(trifluorometil)benzaldehit'in kondensasyonundan elde edilen 4amino-N'-[(4-sübstitüe fenil) metilen]benzoik asit hidrazitinin sübstitüe aril izotiyosiyanatlara katımı ile sentezlendi. Sentezlenen tüm bileşiklerin in vitro olarak MT-4 hücre kültüründe HIV-1 (IIIB) ve HIV-2 (ROD) suşlarına karşı, HeLa, Vero, HEL ve E6SM hücre kültürü ortamlarında HSV-1, HSV-2, Coxsackie B4, Sindbis ve varicella-zoster virüslerine karşı antiviral etkinlikleri ; Mycobacterium tuberculosis H37 Rv suşuna karşı ise antimikobakteriyel etkinlikleri değerlendirilmiştir. Sözkonusu bileşiklerin antikanser ve sitotoksik etki taramaları A 549 and L 929 hücre kültürü ortamlarında belirlenmiştir.

ANAHTAR KELIMELER: Hidrazon, Tiyoüre, Antiviral aktivite, Antikanser aktivite, Mycobacterium tuberculosis H37Rv

\section{REFERENCES}

1. Bermudez LE, Reynolds $R$, Kolonoski $P$, Aralar $P$, Inderlied CB, Young LS. Thiosemicarbazone (thiacetazonelike) compound with activity against Mycobacterium avium in mice. Antimicrob Agents Chemother, 47: 26852687, 2003

2. Phetsuksiri $B$, Baulard $A R$, Cooper $A M$, Minnikin DE, Douglas JD, Besra GS, Brennan PJ. Antimycobacterial activities of isoxyl and new derivatives through the inhibition of mycolic acid synthesis. Antimicrob Agents, Chemother. 43:1042-1051,1999.

3. Liav A, Angala SK, Brennan PJ, Jackson M. N-D-aldopentofuranosyl- $\mathrm{N}^{\prime}$-[p-(isoamyloxy)phenyl]-thiourea derivatives: potential anti-TB therapeutic agents. Bioorg Med Chem Lett, 18:2649-2651, 2008.

4. Sliva K, Schnierle B. From actually toxic to highly specific--novel drugs against poxviruses. Virol J. 4:8, 2007.

5. Patel JM, Dave M, Langalia NA, Thaker KA. Studies antitubercular and antibacterial agents: Preparation of 1-(4aminobenzoyl)-2-benzalhydrazine and 1-[4-(phenylthio ureido)benzoyl]-2-substituted benzalhydrazine. J Indian Chem Soc, 61: 718-720, 1984.

6. Sriram D, Yogeeswari $\mathrm{P}$, Madhu K. Synthesis and in vitro antitubercular activity of some 1-[(4-sub)phenyl]-3-(4-\{1[(pyridine-4-carbonyl)hydrazono]ethyl\}phenyl) thiourea. Bioorg Med Chem Lett, 16: 876-878 , 2006.

7. Rollas S, Küçükgüzel ŞG. Biological Activities of Hydrazone Derivatives. Molecules, 12:1910-1939, 2007.

8. Karakuş S, Küçükgüzel ŞG, Küçükgüzel İ, De Clercq E, Pannecouque C, Andrei G, Snoeck R, Şahin F, Bayrak ÖF. Synthesis, antiviral and anticancer activity of some novel thioureas derived from N-(4-nitro-2-phenoxyphenyl)methanesulfonamide. Eur J Med Chem, 44:3591-3595, 2009.

9. Sriram D,Yogeeswari P,Dinakaran M,Thirumurugan R. Antimycobacterial activity of novel 1-(5-cyclobutyl-1,3oxazol-2-yl)-3-(sub)phenyl/pyridylthiourea compounds endowed with high activity toward multidrug-resistant Mycobacterium tuberculosis. J Antimicrob Chem, 59: 1194-1196, 2007.

10. Kaymakçıŏlu BK, Oruç EE, Ünsalan S, Kandemirli F, Shvets N, Rollas S, Anatholy D. Synthesis and characteri- zation of novel hydrazide-hydrazones and the study of their structure-antituberculosis activity. Eur J Med Chem, 41:1253-1261, 2006.

11. Dixit PP, Patil VJ, Nair PS, Jain S, Sinha N, Arora SK. Synthesis of 1-[3-(4-benzotriazol-1/2-yl-3-fluoro-phenyl)2-oxo-oxazolidin-5-ylmethyl]-3-substituted thiourea derivatives as antituberculosis agents. Eur J Med Chem, 41: 423-428, 2006.

12. Nair PC, Sobhia ME. Quantitative structure activity relationship studies on thiourea analogues as influenza virus neuraminidase inhibitors. Eur J Med Chem, 43: 293-299, 2008.

13. Küçükgüzel İ, Tatar E, Küçükgüzel ŞG, Rollas S, De Clercq E. Synthesis of Some novel thiourea derivatives obtained from 5-[(4-aminophenoxy)methyl]-4-methyl-2,4-dihydro3H-1,2,4-triazole-3-thiones and evaluation as antiviral / anti-HIV agents. Eur J Med Chem, 43: 381-392, 2008.

14. Küçükgüzel ŞG, Rollas $S$, Küçükgüzel İ, Kiraz M. Synthesis and Antimycobacterial activity of some coupling products from 4-aminobenzoic acid hydrazones. Eur J Med Chem, 34: 1093-1100, 1999.

15. Küçükgüzel İ, Küçükgüzel ŞG, Rollas S, Kiraz M. Some 3thioxo / alkylthio-1,2,4-triazoles with a substituted thiourea moiety as possible antimycobacterials. Bioorg Med Chem Lett, 11: 1703-1707, 2001.

16. Karakuş $S$, Rollas $S$. Synthesis and antituberculosis activity of new N-phenyl-N'-[4-(5-alkyl/arylamino-1,3,4-thiadiazole-2-yl)phenyl]thioureas. Farmaco, 57: 577-581, 2002.

17. Kömürcü ŞG, Rollas $S$, Ülgen $M$, Gorrod JW, Çevikbaş A. Evaluation of some arylhydrazones of p-Aminobenzoic acid hydrazide as antimicrobial agents and their in-vitro hepatic microsomal metabolism. Boll Chim Farmaceutico, 134: 375-379, 1995.

18. Rollas $S$, Büyüktimkin $S$, Çevikbaş A. N-[4-(3H-1,3,4oxadiazoline-2-thion-5-yl)phenyl]-N'-substituted thioureas: synthesis and antimicrobiological activities. Arch Pharm (Weinheim), 324:189-190, 1991.

19. Ram VJ, Vidyottama D, Pieters LAC, Vlietinck AJ. J Heterocycl Chem, 26: 625-628, 1989.

20. Kubota S, Horie K, Misra HK, Toyooka K, Uda M, Shibuya M, Terada H. Synthesis and uncoupling activities of hydrophobic thioureas. Chem Pharm Bull, 33: 662-666, 1985. 
21. Koçyiğit-Kaymakçığlu B, Rollas S, Körceğiz E, Arıcıŏ̆lu F. Synthesis and biological evaluation of new N-substituted-N'-(3,5-di/1,3,5-trimethylpyrazole-4-yl)thiourea/ urea derivatives. Eur J Pharm Sci, 26: 97-103, 2005.

22. Küçükgüzel ŞG, Mazi A, Şahin F, Öztürk $S$, Stables JP. Synthesis and biological activities of diflunisal hydrazide-hydrazones. Eur J Med Chem, 38: 1005-1013, 2003.

23. Pauwels R, Balzarini J, Baba M, Snoeck R, Schols D, Herdewijn P, Desmyter J, De Clercq E. Rapid and automated tetrazolium-based colorimetric assay for the detection of anti-HIV compounds. J Virol Methods, 20: 309-321, 1988.

24. Popovic M, Sarngadharan MG, Read E, Gallo RC. Detection, isolation, and continuous production of cytopathic retroviruses (HTLV-III) from patients with AIDS and pre-AIDS. Science, 224: 497-500, 1984.

25. Barré-Sinoussi $F$, Chermann JC, Nugeyre MT, Chamaret S, Grest J, Dauget C, Axler-Blin C, Vézinet-Brun F, Rouz- ioux C, Rozenbaum W, Montagnier L. Science, 220: 868871, 1983.

26. Miyoshi I, Taguchi H, Kobonishi I, Yoshimoto S, Ohtsuki Y, Shiraishi Y, Akagi T. Type C virus-producing cell lines derived from adult T cellleukemia. Gann. Monogr, 28: $219-228,1982$.

27. De Clercq E, Descamps J, Verhelst G, Walker RT, Jones AS, Torrence PF, Shugar D. Comparative efficacy of antiherpes drugs against different strains of herpes simplex virus. J Infect Dis, 141: 563-574, 1980.

28. http://www.taacf.org/about-TAACF.htm

29. Collins L, Franzblau G. Microplate alamar blue assay versus BACTEC 460 system for high-throughput screening of compounds against Mycobacterium tuberculosis and Mycobacterium avium. Antimicrob Agents Chemother, 41:1004-1009, 1997.

30. Ding WQ, Liu B, Vaught JL, Yamauchi H, Lind SE. Anticancer activity of the antibiotic clioquinol. Cancer Res, 65:3389-3395, 2005. 